\title{
Deformations of $G$-structures and infinitesimal automorphisms
}

\author{
By \\ Toshimasa YAGYU \\ (Received October 26, 1971) ${ }^{1)}$
}

When we consider a transitive $G$-structure $g_{0}$ on a compact differentiable manifold $M$, another $G$-structure $g$ on $M$ is said to be locally equivalent to $g_{0}$, if there exists a local transformation $f$ of a neighborhood $U$ of each point of $M$ such that the $G$-structure induced by $f$ from $g_{0}$ is equal to $g$ on $U$, and $g$ is said to be globally equivalent to $g_{0}$, if there exists a global transformation $f$ of $M$ such that the $G$-structure induced by $f$ from $g_{0}$ is equal to $g$ on $M$. The theory of deformations of $G$ structures is considered to represent a difference between the local equivalence and the global equivalence of $G$-structures. In our paper, we take note of a certain global property for $G$-structures and we consider the extent of $G$-structures which are locally equivalent to $g_{0}$ and have the global property. We represent the extent in the space of $G$ structures, using the theory of deformations, and describe a relation between the global property and the equivalence of $G$-structures.

We suppose throughout our paper that $G$ is closed and of finite type and the transitive $G$-structure $g_{0}$ satisfies the following condition. When $\tilde{g}_{0}$ denotes the lift of $g_{0}$ by $p$ on the universal covering manifold $\widetilde{M}$ of $M$, where $p$ is the covering projection, and $\mathfrak{U}\left(\tilde{g}_{0}\right)$ denotes the sheaf of germs of infinitesimal automorphisms of $\tilde{g}_{0}$, the Lie algebra of the Lie group of automorphisms of $\tilde{g}_{0}$ is equal to $H^{0}\left(M, \mathfrak{A}\left(\tilde{g}_{0}\right)\right)$.

1) Revised December 16, 1971 
Then the $G$-structures on $M$ correspond one-to-one to the crosssections of the associated bundle $F(M) / G$ of the frame bundle $F(M)$ over $M$. The set of all $G$-structures forms a Banach manifold $\mathcal{G}$ as the space of cross-sections with respect to a riemannian metric on the bundle of jets of cross-sections. We regard the whole set $\mathscr{D}$ of $G$ structures locally equivalent to $g_{0}$ as a subspace of $\mathscr{G}$. Then deformations of $g_{0}$ are given by curves in $\mathscr{D}$ through $g_{0}$. Let us take note of the equivalence of the infinitesimal automorphisms as a global property. We also regard the whole set $\mathcal{I}$ of $G$-structures having the infinitesimal automorphisms equivalent to those of $g_{0}$ as a subspace of $\mathcal{G}$. A deformation $g_{t}$ of $g_{0}$ is said to have the equivalent infinitesimal automorphisms, if each $G$-structure of $g_{t}$ has the infinitesimal automorphisms equivalent to those of $g_{0}$, that is, if $g_{t}$ is a curve in $\mathcal{I} \cap \mathscr{D}$ through $g_{0}$. Let $\mathcal{E}$ be the subspace of $\mathcal{G}$ consisting of $G$-structures globally equivalent to $g_{0}$ and $\mathcal{S}$ be one consisting of $G$-structures having the same infinitesimal automorphisms as $g_{0}$. The group $\operatorname{Diff}(M)$ of diffeomorphisms of $M$ is a transformation group of $\mathcal{G}$, under which $\mathcal{E}$ and $\mathcal{G}$ are the obrits of $g_{0}$ and $\mathcal{S}$ respectively. Then there exists a differentiable submanifold $\mathscr{V}$ of $\mathscr{G}$ such that $\mathscr{I} \cap \mathscr{D}$ in a neighborhood $U_{g_{0}}$ of $g_{0}$ is the image of $\mathcal{V}$ transformed by the elements of a neighborhood $U_{e}$ of $e$ in $\operatorname{Diff}(M)$. The tangent space of $\mathcal{V}$ at $g_{0}$ is isomorphic to some subspace $\mathcal{K}$ of the kernel of the homomorphism $\omega$ : $H^{1}\left(M, \mathfrak{A}\left(g_{0}\right)\right) \rightarrow H^{1}(M, \mathfrak{R})$ induced by the injection $\mathfrak{X}\left(g_{0}\right) \rightarrow \mathfrak{N}$, where $\mathfrak{A}\left(g_{0}\right)$ is the sheaf of germs of infinitesimal automorphisms of $g_{0}$ and $\mathfrak{N}$ is the sheaf of normalizer of $\mathfrak{A}\left(g_{0}\right)$ in the sheaf of germs of vector fields on $M$. Thus $G$-structures not globally equivalent to $g_{0}$ with respect to the elements of $U_{e}$, but locally equivalent to $g_{0}$ and having the infinitesimal automorphisms equivalent to those of $g_{0}$, exist in $U_{g_{0}}$ to the extent of $\mathcal{K}$. As for deformations of $g_{0}$, infinitesimal deformations corresponding to elements of $\mathcal{K}$ can be extended to deformations having the equivalent infinitesimal automorphisms, and classes of germs of deformations having the equivalent infinitesimal automorphisms are represented uniquely by curves in $\mathcal{V}$ through $g_{0}$. Then we have the 
following proposition as a special case. If the homomorphism $\omega$ is injective, deformations having the equivalent infinitesimal automorphisms are trivial.

\section{$\S 1$. The space of $G$-structures and the group of diffeomorphisms.}

Let $M$ be a compact differentiable manifold of class $C^{\infty}$ with dimension $n$. $G$-structures on $M$ are reductions of the structure group of the frame bundle $F(M)$ over $M$ to a subgroup $G$ of $G L(n)$, where we suppose $G$ to be closed and of finite type. They are represented as submanifolds $B_{G}(M)$ of $F(M)$. Let $F(M) / G$ be the quotient space of $F(M)$ by $G$. Then $G$-structures are represented as cross-sections of $F(M) / G$, where the image of $B_{G}(M)$ by the quotient projection $\pi^{\prime}$ of $F(M)$ onto $F(M) / G$ is the corresponding cross-section of $F(M) / G$. In our paper, we represent $G$-structures by not only submanifolds but cross-sections.

Remark about the class of differentiable G-structures. If the class of differentiable $G$-structures is $C^{r}, B_{G}(M)$ is of class $C^{r}$ and the $s$-th prolongation of $B_{G}(M)$ is of class $C^{r-s}$. Then we take $r>k$, in order that the $k$-th prolongation of $B_{G}(M)$ with $\{e\}$-structure may be of class $C^{1}$, where $k$ is the order of $G$ of finite type. Moreover, we suppose $r$ to be finite.

Let $B_{V}$ be a finite dimensional vector bundle over $M$ of class $C^{r}$. The whole of $r$-jets of cross-sections of $B_{V}$ is a vector bundle over $M$ which is denoted by $B_{V}^{r}$. We define a norm on each fibre of $B_{V}^{r}$ which is continuously dependent to $x \in M$, that is, $\left\|\phi^{r}(x)\right\|$ is continuous on $x$ for any continuous local cross-section $\phi^{r}$ of $B^{r}$. Let us define a norm $\|\phi\|^{(r)}$ of $C^{r}$-cross-section $\phi$ of $B_{V}$ by $\underset{x \in M}{\operatorname{Max}}\left\|j_{x}^{r} \phi\right\|$, where $j_{x}^{r} \phi$ is the $r$-jet of $\phi$ at $x$ and $\left\|j_{x}^{r} \phi\right\|$ is the norm of $j_{x}^{r} \phi$ in the fibre $B_{V}^{r}(x)$ of $B_{V}^{r}$ over $x$. Then the whole of $C^{r}$-cross-sections of $B_{V}$ is a Banach space with respect to the above norm. Let us denote this space by $\Gamma^{(r)}\left(B_{V}\right)$. 
Lemma 1. Let $B_{V}$ and $B_{W}$ be vector bundles of class $C^{r}$ over $M$ and $\eta: B_{V} \rightarrow B_{W}$ be a fibre mapping of class $C^{r}$ such that $\eta$ is infinitely partial differentiable with respect to the fibre of $B_{V}$, every partial derivative of $\eta$ of any order with respect to the fibre is also of class $C^{r}$ and the diffeomorphism of $M$ induced by $\eta$ is identity. Then, the mapping $\bar{\eta}: \Gamma^{(r)}\left(B_{V}\right) \rightarrow \Gamma^{(r)}\left(B_{W}\right)$ defined by $(\bar{\eta} \phi)(x)=\eta(\phi(x))$ is of class $C^{\infty}$.

Proof. $\eta$ induces the continuous mapping $\eta^{r}$ of $B_{V}^{r}$ in $B_{W}^{r}$ well defined by $\eta^{r}\left(j_{x}^{r} \phi\right)=j_{x}^{r}(\bar{\eta} \phi)$ for any $j_{x}^{r} \phi \in B_{V}^{r}$. Then $\eta^{r}$ is infinitely partial differentiable with respect to the fibre and every partial derivative of $\eta^{r}$ of any order with respect to the fibre is continuous on $B_{V}^{r}$. Let $\phi_{0}$ be a fixed element of $\Gamma^{(r)}\left(B_{V}\right)$. We have

$$
\left\|\eta^{r}\left(j_{x}^{r} \phi+j_{x}^{r} \phi_{0}\right)-\eta^{r}\left(j_{x}^{r} \phi_{0}\right)-d \eta_{j_{x}{ }^{\prime} \phi_{0}}^{r}\left(j_{x}^{r} \phi\right)\right\|<\left\|j_{x}^{r} \phi\right\|^{2} \cdot K
$$

for any element $\phi$ of $\Gamma^{(r)}\left(B_{V}\right)$ such that $\|\phi\|^{(r)}<\epsilon$ for a fixed $\epsilon$, where $K$ is a constant independent to $x$ and $d \eta_{j_{x}^{r} \phi_{0}}^{r}$ is the partial differential of $\eta^{r}$ at $j_{x}^{r} \phi_{0}$ with respect to the fibre of $B_{V}^{r}$, because every 2 nd partial derivative of $\eta^{r}$ with respect to the fibre of $B_{V}^{r}$ is bounded on an open set $\bigcup_{x \in M}\left\{j_{x}^{r} \phi \in B_{V}^{r}(x) ;\left\|j_{x}^{r} \phi-j_{x}^{r} \phi_{0}\right\|<\epsilon\right\}$ of $B_{V}^{r}$. Let $\overline{d \eta}_{\phi_{0}}$ be a continuous linear mapping of $\Gamma^{(r)}\left(B_{V}\right)$ into $\Gamma^{(r)}\left(B_{W}\right)$ defined by $\overline{d \eta}_{\phi_{0}}(\phi)(x)=d \eta_{\phi_{0}} \cdot(\phi(x))$, where $d \eta_{\phi_{0}}: B_{V} \rightarrow B_{W}$ is the partial differential of $\eta$ at $\phi_{0}$ with respect to the fibre. Since the mapping of $B_{V}^{r}$ into $B_{W}^{r}$ induced by $d \eta_{\phi_{0}}$ is $d \eta_{j_{x}^{r} \phi_{0}}^{r}$ over $x$, we have

$$
\begin{aligned}
& \left\|\bar{\eta}\left(\phi_{0}+\phi\right)-\bar{\eta}\left(\phi_{0}\right)-\overline{d \eta}_{\phi}(\phi)\right\|^{(r)} \\
& \quad=\operatorname{Max}_{x \in M}\left\|j_{x}^{r}\left(\bar{\eta}\left(\phi_{0}+\phi\right)-\bar{\eta}\left(\phi_{0}\right)-d \bar{\eta}_{\phi}(\phi)\right)\right\| \\
& \quad=\operatorname{Max}_{x \in M}\left\|\eta^{r}\left(j_{x}^{r}\left(\phi_{0}+\phi\right)\right)-\eta^{r}\left(j_{x}^{r}\left(\phi_{0}\right)\right)-d \eta_{j_{x}^{r} \phi_{0}}^{r}\left(j_{x}^{r} \phi\right)\right\| \\
& \quad<\operatorname{Max}_{x \in M}\left\|j_{x}^{r} \phi\right\|^{2} \cdot K \\
& \quad=\left(\|\phi\|^{(r)}\right)^{2} \cdot K, \text { for }\|\phi\|^{(r)}<\epsilon
\end{aligned}
$$


Therefore, we have $\lim _{\|\phi\| \rightarrow 0}\left\|\bar{\eta}\left(\phi_{0}+\phi\right)-\bar{\eta}\left(\phi_{0}\right)-\overline{d \eta}_{\phi_{0}}(\phi)\right\|^{(r)} /\|\phi\|^{(r)}=0$, that is, $\bar{\eta}$ is differentiable at $\phi_{0}$. Next, we consider the bundle Hom $\left(B_{V} ; B_{W}\right)$ of which the fibre over each $x$ is a linear space of homomorphisms of $B_{V}(x)$ into $B_{W}(x)$. The bundle $\mathrm{Hom}^{r}\left(B_{V} ; B_{W}\right)$ of $r$-jets of cross-sections of Hom $\left(B_{V} ; B_{W}\right)$ can be identified with a subbundle of the bundle $\operatorname{Hom}\left(B_{V}^{r}: B_{V V}^{r}\right)$, of which each fibre has a norm continuously dependent to $x \in M$ defined by the norm of $B_{V}^{r}(x)$ and that of $B_{W}^{r}(x)$. The space $\Gamma^{(r)}\left(\operatorname{Hom}\left(B_{V} ; B_{W}\right)\right)$ with respect to the above norm can be identified with a subspace of the Banach space $L\left(\Gamma^{(r)}\right.$ $\left.\left(B_{V}\right) ; \Gamma^{(r)}\left(B_{W}\right)\right)$ of continuous linear mapping of $\Gamma^{(r)}\left(B_{V}\right)$ into $\Gamma^{(r)}$ $\left(B_{W}\right)$. Let $d \eta$ be the partial derivative of $\eta$ with respect to the fibre of $B_{V}$ and then it is a fibre mapping of $B_{V}$ into $\operatorname{Hom}\left(B_{V} ; B_{W}\right)$. If we take the bundle Hom $\left(B_{V} ; B_{W}\right)$ instead of $B_{W}$, the mapping $d \eta$ satisfies the condition of $\eta$ in Lemma 1 and we have a differentiable mapping

$$
\overline{d \eta}: \Gamma^{(r)}\left(B_{V}\right) \longrightarrow \Gamma^{(r)}\left(\operatorname{Hom}\left(B_{V} ; B_{W}\right)\right) \subset L\left(\Gamma^{(r)}\left(B_{V}\right) ; \Gamma^{(r)}\left(B_{W}\right)\right)
$$

induced from $d \eta$, such that $\overline{d \eta}\left(\phi_{0}\right)$ for any $\phi_{0} \in \Gamma^{(r)}\left(B_{V}\right)$ is the differential of $\bar{\eta}$ at $\phi_{0}$. Following the above argument for any order of the differential of $\bar{\eta}$ in succession, we conclude the mapping $\bar{\eta}$ is of class $C^{\infty}$.

Remark. Even if $\eta$ is not a mapping of the whole space of $B_{V}$ into $B_{W}$ but a mapping of a fibre subspace $B^{\prime}$ of $B_{V}$ into $B_{W}$, Lemma 1 is right for $\Gamma^{(r)}\left(B^{\prime}\right)$ instead of $\Gamma^{(r)}\left(B_{V}\right)$.

Let $B$ be a fibre bundle over $M$ of class $C^{\infty}$ and let us define a riemannian metric on $B$ of class $C^{\infty}$. Let $B^{r}$ denote a bundle of $r$-jets of cross-sections of $B$. Since $B^{r}$ is a $C^{\infty}$-bundle over $B$, we can define a riemannian metric on $B^{r}$ of class $C^{\infty}$ based on the metric on $B$ such that $\rho_{x}\left(\pi^{r} b, \pi^{r} b^{\prime}\right) \leqq \rho_{x}^{r}\left(b, b^{\prime}\right)$ for each $x$, where $b, b^{\prime} \in B^{r}(x), \pi^{r}: B^{r} \rightarrow B$ is the canonical projection and $\rho_{x}$ (resp. $\rho_{x}^{r}$ ) is the distance along each fibre $B(x)\left(\right.$ resp. $\left.B^{r}(x)\right)$. Let $\Gamma^{(r)}(B)$ be the whole of $C^{r}$-cross-sections 
of $B$ with the metric defined by

$$
\rho^{(r)}(\phi, \psi)=\operatorname{Max}_{x \in M} \rho_{x}^{r}\left(j_{x}^{r} \phi, j_{x}^{r} \psi\right) \text { for } \phi, \psi \in \Gamma^{(r)}(B) .
$$

Applying the notion of the Banach manifold (see [2]) to $\Gamma^{(r)}(B)$, under the fact of Lemma 1 which gives the smoothness of the coordinate transformation, we have

Proposition 1. The metric space $\Gamma^{(r)}(B)$ is a Banach manifold of class $C^{\infty}$. The tangent space of $\Gamma^{(r)}(B)$ at $\phi$ is the Banach space $\Gamma^{(r)}\left(V_{\phi}(B)\right)$, where $V_{\phi}(B)$ is the bundle of vertical vectors of $B$ at $\phi$.

Definition. The space $\mathcal{G}$ of $G$-structures of class $C^{r}$ on $M$ is the Banach manifold $\Gamma^{(r)}(F(M) / G)$ of class $C^{\infty}$, with respect to a riemannian metric of the bundle space of $r$-jets of cross-sections of $F(M) / G$.

The tangent space $T_{g}(\mathcal{G})$ at $g \in \mathcal{G}$ is the Banach space $\Gamma^{(r)}$ $\left(V_{g}(F(M) / G)\right)$, where $V_{g}(F(M) / G)$ is the vertical vector bundle of $F(M) / G$ at $g$.

Let $B$ and $B^{\prime}$ be fibre bundles of class $C^{\infty}$ over $M$ and $\xi: B \rightarrow B^{\prime}$ be a fibre mapping of class $C^{r}$ such that $\xi$ is infinitely partial differentiable with respect to the fibre of $B$, every partial derivative of any order of $\xi$ with respect to the fibre is of class $C^{r}$ and the diffeomorphism of $M$ induced by $\xi$ is identity. Let us define the mapping $\bar{\xi}: \Gamma^{(r)}(B)$ $\rightarrow \Gamma^{(r)}\left(B^{\prime}\right)$ by $(\bar{\xi} \phi)(x)=\xi(\phi(x))$. Since $\xi$ induces a mapping $\dot{\bar{\xi}}$ of the tangent space $\Gamma^{(r)}\left(V_{\phi}(B)\right)$ to $\Gamma^{(r)}\left(V_{\psi}\left(B^{\prime}\right)\right)$ for any $\xi \in \Gamma^{(r)}(B), \psi \in$ $\Gamma^{(r)}\left(B^{\prime}\right)$ and $\dot{\bar{\xi}}$ is of class $C^{\infty}$ by Lemma 1 , we have

Proposition 2. The mapping $\bar{\xi}$ is of class $C^{\infty}$.

Let us define a riemannian metric of class $C^{\infty}$ on $M$. The product mainfold $M \times M$ is a trivial bundle over $M$ and the space $C^{\left(r^{\prime}\right)}(M)$ of $C^{r^{\prime}}$-transformations of $M$ is the Banach manifold $\Gamma^{\left(r^{\prime}\right)}(M \times M)$ of $C^{r^{\prime} \text { - }}$ 
cross-sections of the above bundle with respect to a riemannian metric of the bundle $J^{r^{\prime}}(M \times M)$ of $r^{\prime}$-jets based on the product riemannian metric of $M \times M$. Any element of the $\epsilon$-neighborhood of identity of $C^{\left(r^{\prime}\right)}(M)$ is a $C^{r^{\prime}}$-diffeomorphism of $M$ by the definition of the metric of $C^{\left(r^{\prime}\right)}(M)$ and then the set of $C^{r^{\prime}}$-diffeomorphisms of $M$ is an open subspace of $C^{\left(r^{\prime}\right)}(M)$. Let $\rho^{\left(r^{\prime}\right)}$ be the metric of $C^{\left(r^{\prime}\right)}(M)$. We define the metric $\rho\left(f_{1}, f_{2}\right)=\rho^{\left(r^{\prime}\right)}\left(f_{1}, f_{2}\right)$ on the set $\operatorname{Diff}{ }^{\left(r^{\prime}\right)}(M)$ of $C^{r^{\prime}}$-diffeomorphisms of $M$. Then $\operatorname{Diff}^{\left(r^{\prime}\right)}(M)$ is a Banach manifold of class $C^{\infty}$. The tangent space of Diff ${ }^{\left(r^{\prime}\right)}(M)$ at any $f$ is the Banach space $\Gamma^{\left(r^{\prime}\right)}(T(M))$ with respect to the norm of each fibre of $J^{r^{\prime}}(T(M))$ based on the metric of $J^{r^{\prime}}(M \times M)$, where $T(M)$ is the tangent bundle which is identified with the vertical vector bundle of the trivial bundle $M \times M$ at $f$.

\section{§. Infinitesimal automorphisms.}

Let $\theta$ be a vector field of class $C^{r+1}$ on an open set $U$ of $M$. For $g \in \mathcal{G}$, let $g^{\prime}$ be a cross-section of $F(M)$ on $U$ such that $\pi^{\prime} g^{\prime}=g$ and $\mathcal{L}_{\theta} g^{\prime}$ be the Lie derivative of a tensor field $g^{\prime}$ with respect to $\theta$. If we set $\mathcal{L}_{\theta} g^{\prime}=g^{\prime} \times \mathfrak{a}$, then $\mathfrak{a}$ is a $\mathfrak{g l}$-valued function on $U$. Since $F(M) \times \mathfrak{g l}$ is the bundle of vertical vectors of $F(M), g^{\prime} \times \mathfrak{a}$ is a vertical vector field of $F(M)$ at $g^{\prime}$. The bundle of vertical vectors of $F(M) / G$ is an associated bundle $F(M) \times \underset{G}{f}$ of $F(M)$ by the linear isotropy representation is: $G \rightarrow G L(\mathfrak{f})$, where $\mathrm{f}=\mathrm{gl} / \mathrm{g}$. Then $g^{\prime} \underset{G}{\times} q \cdot \mathfrak{a}$ is a vertical vector field of $F(M) / G$ at $g$, where $q$ is the projection $\mathfrak{g l} \rightarrow \mathfrak{f}$. This field is determined by $\theta$ and $g$, that is, $g^{\prime} \underset{G}{\times} q \cdot a$ is independent to a choice of $g^{\prime}$ such that $\pi^{\prime} g^{\prime}=g$. We denote $g^{\prime} \underset{G}{\times} q \cdot a$ by $\mathcal{L}_{\theta} g$. Then $\theta$ is an infinitesimal automorphism of $g$, if and only if $\mathcal{L}_{\theta} g=0$.

By the condition of $g_{0}$ in Introduction, $g_{0}$ is of class $C^{\infty}$. When $\theta$ is a global vector field of class $C^{r+1}$ on $M, \mathcal{L}_{\theta} g_{0}$ is a global $C^{r}$-crosssection of the vertical vector bundle $V_{g_{0}}(F(M) / G)$ of $F(M) / G$ at $g_{0}$. Then we have a linear mapping $\bar{\delta}_{g_{0}}$ of the Banach space $\Gamma^{(r+1)}(T(M))$ of all vector fields of class $C^{r+1}$ on $M$ into the Banach space $\Gamma^{(r)}\left(V_{g_{0}}\right.$ $(F(M) / G))$ of all $C^{r}$-cross-sections of $V_{g_{0}}(F(M) / G)$, such that $\bar{\delta}_{g_{0}} \theta=$ $\mathcal{L}_{\theta} g_{0}$. 
Proposition 3. The linear mapping $\bar{\delta}_{g_{0}}$ is continuous.

Proof. Since a vertical vector $\mathcal{L}_{\theta} g_{0}(x)$ is determined by $j_{x}^{1} \theta$ and $j^{1} g_{0}$, we have a mapping $L$ of the bundle $J^{1}(T(M))$ of 1 -jets of vector fields on $M$ into $V_{g_{0}}(F(M) / G)$ such that $L\left(j_{x}^{1} \theta\right)=\mathcal{L}_{\theta} g_{0}$ for any vector field $\theta$ on a neighborhood of $x$, and $L$ is a bundle mapping of vector bundles. Then $L$ induces a continuous linear mapping $\bar{L}$ of $\Gamma^{(r)}$ $\left(J^{1}(T(M))\right)$ into $\Gamma^{(r)}\left(V_{g_{0}}(F(M) / G)\right)$ and a correspondence defined by $\theta \rightarrow j^{1} \theta$ is an imbedding im of $\Gamma^{(r+1)}(T(M))$ into $\Gamma^{(r)}\left(J^{1}\right.$ $(T(M)))$. Then $\bar{L} \cdot i m$ is continuous linear and $\bar{\delta}_{g_{0}}=\bar{L} \cdot i m$.

Taking the germs of each cross-section, the correspondence $\theta \rightarrow$ $\mathcal{L}_{\theta} g_{0}$ induces a sheaf homomorphism $\delta_{g_{0}}: \mathfrak{I} \rightarrow \mathfrak{B}$, where $\mathfrak{I}$ is a sheaf of germs of vector fields of class $C^{r+1}$ on $M$ and $\mathfrak{B}$ is a sheaf of germs of $C^{r}$-cross-sections of $V_{g_{0}}(F(M) / G)$. The kernel of $\delta_{g_{0}}$ is the sheaf $\mathfrak{A}\left(g_{0}\right)$ of germs of infinitesimal automorphisms of $g_{0}$. Since $G$ is of finite type, the sheaf $\mathfrak{A}\left(g_{0}\right)$ is locally constant and its stalks are finite dimensional vector spaces. Then the set $\Gamma\left(\mathfrak{A}\left(g_{0}\right)\right)\left(=H^{0}\left(M, \mathfrak{A}\left(g_{0}\right)\right)\right)$ of global infinitesimal automorphisms is a finite dimensional vector space which is a subspace of $\Gamma^{(r+1)}(T(M))$. Thus we have a closed complement $D$ of $\Gamma\left(\mathfrak{U}\left(g_{0}\right)\right)$ in $\Gamma^{(r+1)}(T(M))$ and $\bar{\delta}_{g_{0}}$ is isomorphic on $D$.

A $C^{r+1}$-diffeomorphism $f$ of $M$ induces a $C^{r}$-diffeomorphism $f^{\prime}$ of $F(M)$ such that $f^{\prime}(b \cdot a)=\left(f^{\prime}(b)\right) \cdot a$ for any $a \in G$ and $b \in F(M)$, and then it induces a $C^{r}$-diffeomorphism $f^{*}$ of $F(M) / G$ such that $\pi^{\prime}\left(f^{\prime}(b)\right)$ $=f^{*} \pi^{\prime}(b)$ and $f\left(\pi\left(b^{\prime}\right)\right)=\pi\left(f^{*}\left(b^{\prime}\right)\right)$ where $b^{\prime} \in F(M) / G$. Let us define $\bar{f} g$ by $(\bar{f} g)(x)=f^{*-1}(g(f(x)))$ for any $g \in \mathcal{G}$. Then $\bar{f} g$ is a new $C^{r}$ cross-section of $F(M) / G$ and $\bar{f}$ is a transformation of the space $\mathcal{G}$. The partial differential of $f^{*}$ with respect to the fibre of $F(M) / G$ is a diffeomorphism $f^{* *}$ of the vertical vector bundle defined by $f^{* *} v$

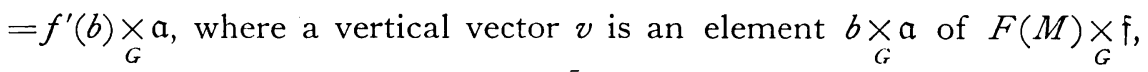
and $f^{* *}$ induces a transformation $\bar{f}^{* *}$ of the vertical vector fields $\bar{v}$ by $\left(\bar{f}^{* *} \bar{v}\right)(x)=f^{* *-1}(\bar{v}(f(x))$. 
Proposition 4. $\bar{f}^{* *}\left(\mathcal{L}_{\theta} g\right)=\mathcal{L}_{f \theta}(\bar{f} g)$.

Proof. If $\pi^{\prime} g^{\prime}=g$ and $\mathcal{L}_{\theta} g^{\prime}=g^{\prime} \times a$, then

$$
\begin{aligned}
\left(\mathcal{L}_{f^{-1}} \theta\left(f^{\prime-1} g^{\prime}\right)\right)(x)= & f^{\prime \prime-1}\left(\mathcal{L}_{\theta} g^{\prime}(f(x))\right) \\
& =f^{\prime \prime-1}\left[g^{\prime}(f(x)) \times \mathfrak{a}(f(x))\right],
\end{aligned}
$$

where $f^{\prime \prime}$ is a diffeomorphism of vertical vector bundle of $F(M)$ induced by $f^{\prime}$. Therefore, we have

$$
\begin{aligned}
{\left[\mathcal{L}_{f \theta}(\bar{f} g)\right](x) } & =f^{\prime \prime-1}\left[g^{\prime}(f(x))\right] \underset{G}{\times} q[\mathfrak{a}(f(x))] \\
& =f^{* *-1}\left[g^{\prime}(f(x)) \underset{G}{\times} q(\mathfrak{a}(f(x))]\right. \\
& =f^{* *-1}\left[\mathcal{L}_{\theta} g(f(x))\right]=\left(\bar{f}^{* *}\left(\mathcal{L}_{\theta} g\right)\right)(x) .
\end{aligned}
$$

\section{§. Transitive $G$-structures and associated $G$-structures.}

In our paper, we suppose that the $G$-structure $g_{0}$ is transitive, that is, the local automorphisms of $g_{0}$ act locally transitive on $M$ and moreover those of every prolongation $B_{G}^{\mu}(\mu)$ of $B_{G}\left(=g_{0}\right)$ act locally transitive on $B_{G}^{\mu}(\mu)$, (see [4], Appendix I).

If and only if $n$ is an element of the normalizer $N$ of $G$ in $G L(n)$, the right translation of $g_{0}$ by $n$ is also a $G$-structure, which is called to be associated to $g_{0}$. By the theory of $G$-structures (see [1]), we have

Proposition 5. A G-structure $g$ is associated to $g_{0}$, if and only if $g$ has the same local infinitesimal automorphisms as $g_{0}$.

The product space $N \times M$ is a trivial $C^{\infty}$-bundle over $M$ and then a mapping of $N \times M$ into $F(M) / G$ defined by $n \times x \rightarrow g_{0}(x) \cdot n$ satisfies the condition of $\xi$ of Proposition 2, because $g_{0}$ is of class $C^{\infty}$. Since $N$ is a closed submanifold of $\Gamma^{(r)}(N \times M)$ as the constant cross-sections, the mapping $\rho_{g_{0}}: N \rightarrow \mathcal{G}$ defined by $\rho_{g_{0}}(n)=g_{0} \cdot n$ is of class $C^{\infty}$ by Proposition 2. For each $x$, the set $\left\{g_{0}(x) \cdot n ; n \in N\right\}$ is a closed submanifold of the fibre of $F(M) / G$ over $x$. Thus we have 
Proposition 6. Let $\mathcal{A}$ be the set of G-structures of class $C^{r}$ associated to $g_{0}$. Then $\mathcal{A}$ is a closed submanifold of $\mathcal{G}$.

If and only if $n \cdot n^{\prime-1} \in G$, we have $\rho_{g_{0}}(n)=\rho_{g_{0}}\left(n^{\prime}\right)$. Then $\rho_{g}$ induces a $C^{\infty}$-imbedding $\bar{\rho}_{g}: N / G \rightarrow \mathcal{G}$ such that $\bar{\rho}_{g} q^{\prime}=\rho_{g}$, where $q^{\prime}: N \rightarrow N / G$.

We consider a mapping of $\operatorname{Diff}^{(r+1)}(M) \times N / G$ into $\mathcal{G}$ defined by $f \times \bar{n} \rightarrow \bar{f} \cdot \bar{\rho}_{g_{0}}(\bar{n})$. Let $J^{1}(M, \alpha)$ (resp. $\left.J^{1}(M, \beta)\right)$ be the fibre bundle of invertible 1-jets of diffeomorphisms of $M$ with the source projection $\alpha($ resp. the target projection $\beta)$ as the bundle projection. Each jet $j^{1} f^{-1}$ with source $y$ and target $x$ operates on the fibre of $F(M) / G$ over $y$ such that $\left(j^{1} f^{-1}\right) \cdot g(y)=(\bar{f} g)(x)$ for each $g \in \mathcal{G}$. The product space $J^{1}(M, \beta) \times N / G$ is also a $C^{\infty}$-bundle over $M$ with the projection $\bar{\beta}$ : $\left(j^{1} f, \bar{n}\right) \rightarrow \beta\left(j^{1} f\right)$. Since $g_{0}$ is of class $C^{\infty}$, the mapping of $J^{1}(M, \beta)$ $\times N / G$ into $F(M) / G$ defined by $\left(j^{1} f^{-1}, \bar{n}\right) \rightarrow\left(j^{1} f^{-1}\right) \cdot\left(\bar{\rho}_{g_{0}}(\bar{n})(y)\right)$ is a fibre mapping of class $C^{\infty}$. Then, by Proposition 2 we have a $C^{\infty}$-mapping $\tau^{\prime}: \Gamma^{(r)}\left(J^{1}(M, \beta) \times N / G\right) \rightarrow \Gamma^{(r)}(F(M) / G)$. The space $\Gamma^{(r)}$ $\left(J^{1}(M, \beta) \times N / G\right)$ is $C^{\infty}$-diffeomorphic to $\Gamma^{(r)}\left(J^{1}(M, \beta)\right) \times \Gamma^{(r)}(M, N / G)$. On the other hand, the correspondence $j_{x}^{1} f \rightarrow j_{y}^{1} f^{-1}$, where $y=f(x)$, gives a $C^{\infty}$-isomorphism of the bundle $J^{1}(M, \alpha)$ onto $J^{1}(M, \beta)$, which induces a $C^{\infty}$-diffeomorphism $\iota: \Gamma^{(r)}\left(J^{1}(M, a)\right) \rightarrow \Gamma^{(r)}\left(J^{1}(M, \beta)\right)$ such that $\iota\left(j^{1} f\right)=j^{1} f^{-1}$. From the definition of the Banach manifold $\operatorname{Diff}^{(r+1)}(M)$ in $\S 1$, we have a $C^{\infty}$-injection $\iota^{\prime}$ of $\operatorname{Diff}{ }^{(r+1)}(M)$ into $\Gamma^{(r)}$ $\left(J^{1}(M, \alpha)\right)$ such that $\iota^{\prime}(f)=j^{1} f$, and we have a $C^{\infty}$-injection $\iota^{\prime \prime}$ of Diff ${ }^{(r+1)}(M)$ into $\Gamma^{(r)}\left(J^{1}(M, \beta)\right)$ such that $\iota^{\prime \prime}(f)=\iota^{\prime} \iota^{\prime}(f)=j^{1} f^{-1}$. The $C^{\infty}$ manifold $N / G$, of which each element can be considered as a constant mapping of $M$ into $N / G$, is a $C^{\infty}$-submanifold of $\Gamma^{(r)}(M, N / G)$ and then we have a $C^{\infty}$-injection $\kappa: N / G \rightarrow \Gamma^{(r)}(M, N / G)$. Let $\tau$ be the composed mapping of

$\tau^{\prime \prime} \times \kappa: \operatorname{Diff}{ }^{(r+1)}(M) \times N / G \rightarrow \Gamma^{(r)}\left(J^{1}(M, \beta)\right) \times \Gamma^{(r)}(M, N / G)$,

the isomorphism: $\Gamma^{(r)}\left(J^{1}(M, \beta)\right) \times \Gamma^{(r)}(M, N / G)$

$$
\rightarrow \Gamma^{(r)}\left(J^{1}(M, \beta) \times N / G\right)
$$


and $\tau^{\prime}: \Gamma^{(r)}\left(J^{1}(M, \beta) \times N / G\right) \rightarrow \Gamma^{(r)}(F(M) / G)$.

Then $\tau$ is of class $C^{\infty}$ from $\operatorname{Diff}{ }^{(r+1)}(M) \times N / G$ into $\mathcal{G}$ such that $\tau(f \times \bar{n})$ $=\bar{f} \bar{\rho}_{g_{0}}(\bar{n})$. By the definition of $\mathcal{L}_{\theta} g_{0}$, the partial differential of $\tau$ at (identity $\times \bar{e}$ ) with respect to $\operatorname{Diff}^{(r+1)}(M)$ is a continuous linear mapping $\bar{\delta}_{g_{0}}$ in Proposition 3. The partial differential of $\tau$ at (identity $\times \bar{e}$ ) with respect to $N / G$ is a continuous linear mapping of $\mathfrak{\imath} / \mathfrak{g}$ into $\Gamma^{(r)}\left(V_{g_{0}}(F(M) /\right.$ $G)$ ) defined by $\dot{n} \rightarrow g_{0}^{\prime} \underset{G}{\times} \dot{n}$, where $g_{0}=\pi^{\prime} g_{0}^{\prime}$. Thus we have

Proposition 7. The mapping $\tau$ is of class $C^{\infty}$. The differential of $\tau$ at (identity $\times \bar{e}$ ) is a continuous linear mapping of $\Gamma^{(r+1)}(T(M)$ ) $\times \mathfrak{n} / \mathfrak{g}$ into $\Gamma^{(r)}\left(V_{g_{0}}(F(M) / G)\right)$ defined by $\theta \times \dot{n} \rightarrow \bar{\delta}_{g_{0}} \theta+\left(g_{0}^{\prime} \times \underset{G}{n}\right)$ where $\pi^{\prime} g_{0}^{\prime}=g_{0}$.

\section{$\S 4$. Transformation of the infinitesimal automorphisms.}

Proposition 8. Let $f$ be a local diffeomorphism of class $C^{r+1}$ with domain $U$. A local isomorphism of the sheaf $\mathfrak{I}$ induced by $f$ maps a potion $\mathfrak{A}\left(g_{0}\right) \mid U$ of $\mathfrak{A}\left(g_{0}\right)$ over $U$ onto $\mathfrak{A}\left(g_{0}\right) \mid f(U)$, if and only if a G-structure $\bar{f} g_{0}$ induced from $g_{0}$ by $f$ has the same infinitesimal automorphisms as $g_{0}$ on $f(U)$.

Proof. By Proposition 4, we have $f\left(\mathfrak{A}\left(g_{0}\right) \mid U\right)=\mathfrak{A}\left(\bar{f} g_{0}\right) \mid f(U)$. Then $\mathfrak{A}\left(g_{0}\right) \mid f(U)=f\left(\mathfrak{U}\left(g_{0}\right) \mid U\right)$, if and only if $\mathfrak{U}\left(g_{0}\right)\left|f(U)=\mathfrak{A}\left(\bar{f} g_{0}\right)\right|$ $f(U)$.

Proposition 9. Let $f(t)$ be the 1-parameter diffeomorphisms $(\exp t \theta)$ generated by a local vector field $\theta$. If and only if each $f(t)$ satisfies the condition of Proposition 8 , the germs of $\theta$ belong to the sheaf $\mathfrak{N}$ of normalizer of $\mathfrak{A}\left(g_{0}\right)$ in $\mathfrak{I}$.

Proof. Let $U$ be an open set of $M$ and $\theta$ be a vector field on $U$. For an open set $V \subset U$, each $f(t)$ is diffeomorphism with domain $V$ for a suitable small interval of $|t|$ such that $f(t) \cdot V \subset U$. If $f(t)\left(\mathfrak{A}\left(g_{0}\right) \mid V\right)$ 
$=\mathfrak{A}\left(g_{0}\right) \mid f(t) V$, then $[\theta, \lambda]$ is an infinitesimal automorphism for any infinitesimal automorphisms $\lambda$ on $V$. Since $V$ is any open set of $U$, the germ of $\theta$ at any $x \in U$ is in $\mathfrak{R}$. Conversely, let $n$ be a vector field on $U$ such that its germs belong to $\mathfrak{R}$. Then $\mathrm{e}^{\mathrm{ad}(t n)}$ is an infinitesimal automorphism on $U$ for any $\lambda$. Local diffeomorphisms (exp $t n) \cdot(\exp s \lambda) \cdot(\exp t n)^{-1}$ for a small fixed $|t|$ are local automorphisms $a(s)$ of $g_{0}$ for a suitable small $|s|$ and on a suitable domain such that the above compositions are considerable, because $(\exp t n) \cdot(\exp s \lambda)$. $(\exp t n)^{-1}=\exp \left(\mathrm{e}^{\operatorname{ad}(t n)} s \lambda\right) . \quad$ By Proposition 4,

$$
\mathcal{L}(\exp t n) \lambda g_{0}=(\exp t n)^{* *} \mathcal{L}_{\lambda}\left((\exp t n)^{-1} g_{0}\right)
$$

On the notation ()$^{\prime}$ in $\S 2$, we have

$$
\begin{gathered}
\mathcal{L}_{\lambda}\left((\overline{\exp t n})^{-1} g_{0}\right)^{\prime}(x)=\left[\frac{\mathrm{d}}{\mathrm{d} s}(\exp s \lambda)^{\prime-1}(\exp t n)^{\prime-1}\right. \\
\left.g_{0}^{\prime}((\exp t n)(\exp s \lambda) x)\right]_{s=0} \\
=\left[\frac{\mathrm{d}}{\mathrm{d} s}(\exp t n)^{\prime-1} a(s)^{\prime-1}\left(g_{0}^{\prime}(a(s)(\exp t n) x)\right)\right]_{s=0} \\
=\left[(\exp t n)^{\prime-1} \frac{\mathrm{d}}{\mathrm{d} s} g^{\prime}(s)((\exp t n) x)\right]_{s=0^{\prime}}
\end{gathered}
$$

where $g^{\prime}(s)=\overline{a(s)^{\prime}-1} g_{0}$. Here $a^{\prime}(s)(y) \in G$, if we set $g^{\prime}(s)(y)=g_{0}^{\prime}(y)$ $\left(a^{\prime}(s)(y)\right)$ and then $\mathrm{a}(y) \in \mathrm{g}$, if we set $\left[\frac{\mathrm{d}}{\mathrm{d} s} g^{\prime}(s)(y)\right]_{s=0}=g_{0}^{\prime}(y) \underset{G}{\times} \mathfrak{a}(y)$. Therefore,

$$
\begin{aligned}
& \mathcal{L}_{\lambda}\left((\overline{\exp t n})^{-1} g_{0}\right)(x) \\
& \quad=(\exp t n)^{* *-1}\left[g_{0}^{\prime}((\exp t n) x) \underset{G}{\times a} \mathrm{a}((\exp t n) x)\right]=0
\end{aligned}
$$

and then $(\exp t n) \lambda$ is a local infinitesimal automorphism.

Proposition 10. The dimension of the stalk of $\mathfrak{N}$ is finite and constant for every $x \in M$.

Proof. For a point $x_{0} \in M$, the adjoint representation of $\mathfrak{R}\left(x_{0}\right)$ 
on $\mathfrak{U}\left(g_{0}\right)\left(x_{0}\right)$ defines a homomorphism $K$ from an additive group $\mathfrak{N}\left(x_{0}\right)$ into an additive group $\operatorname{Hom}\left(\mathfrak{A}\left(g_{0}\right)\left(x_{0}\right)\right)$, where $\mathfrak{N}\left(x_{0}\right)\left(\operatorname{resp} \cdot \mathfrak{A}\left(g_{0}\right)\left(x_{0}\right)\right)$ is a stalk of $\mathfrak{X}\left(\right.$ resp. $\left.\mathfrak{A}\left(g_{0}\right)\right)$ at $x_{0}$. Each element of kernel of $K$ is the germ of vector field $n$ on a neighborhood of $x_{0}$ at $x_{0}$ such that $[n, \lambda]=0$ for any infinitesimal automorphisms $\lambda$ on $U$. Since $g_{0}$ is transitive, there exist $n$ independent infinitesimal automorphisms $\lambda_{i}(i=1, \ldots, n)$ on $U$. The condition $\left[n, \lambda_{i}\right]=0(i=1, \ldots, n)$ is a system $\lambda_{j}\left(n^{i}\right)=$

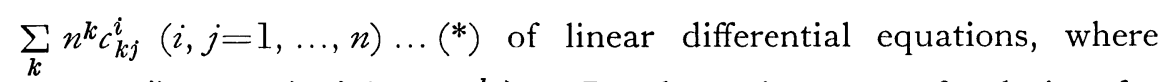
$n=\sum_{i} n^{i} \lambda_{i}$ and $\left[\lambda_{i}, \lambda_{j}\right]=\sum_{k} c_{i j}^{k} \lambda_{k}$. By the uniqueness of solution for the initial condition $n\left(x_{0}\right)$, the dimension of the solutions is finite. Therefore the dimension of kernel of $K$ is finite. Since dim. (Hom $\left.\left(\mathfrak{U}\left(g_{0}\right)\left(x_{0}\right)\right)\right)$ is finite, $\operatorname{dim} . \mathfrak{N}\left(x_{0}\right)$ is finite. Since $g_{0}$ is transitive, there exists a local automorphism $f$ of a neighborhood of $x$ onto that of $x^{\prime}$ for any $x, x^{\prime}$ of $M$ and $f$ induces an isomorphism of $\mathfrak{R}(x)$ onto $\mathfrak{N}\left(x^{\prime}\right)$. Then, $\operatorname{dim} . \mathfrak{R}(x)=\operatorname{dim} . \mathfrak{R}\left(x^{\prime}\right)$.

Proposition 11. The sheaf $\mathfrak{R}$ is locally constant.

Proof. Since $\operatorname{dim} . \mathfrak{N}\left(x_{0}\right)$ is finite, $\mathfrak{N}\left(x_{0}\right)$ is the germs of vector fields $n$ on some common neighborhood $U$ of $x_{0}$ such that $[n, \lambda]$ are infinitesimal automorphisms on $U$ for any infinitesimal automorphisms $\lambda$ on $U$. Let $(\Re, U)$ denote the whole of such vector fields $n$ on $U$ and let $n_{1}, n_{2} \in(\Re, U)$. If $n_{1}=n_{2}$ on an open set $V$ of $U$, then $\left[n_{1}-n_{2}, \lambda\right]$ $=0$ on $U$ for any $\lambda$. Then $n_{1}-n_{2}$ is a solution of the system (*) in Proof of Proposition 10 and then $n_{1}=n_{2}$ on $U$. Therefore each vector field of $(\mathfrak{R}, U)$ has a respectively different germ at any $x \in U$. Since $\operatorname{dim} . \mathfrak{N}(x)$ is constant, the whole of germs of vector fields of $(\mathfrak{N}, U)$ at every point of $U$ is the portion $\mathfrak{N} \mid U$. Therefore $\mathfrak{N}$ is locally constant.

Since the dimension of the space $\Gamma(\mathfrak{N}, M)$ is finite, we have by Palais' theorem ([5])

Proposition 12. Let $N\left(g_{0}\right)$ be the group of $C^{r+1}$-diffeomorphisms of $M$ which map all the local infinitesimal automorphisms of $g_{0}$ onto 
themselves. Then $N\left(g_{0}\right)$ is a Lie group.

Let $\tilde{g}_{0}$ be the lift of $g_{0}$ on the universal covering manifold $\tilde{M}$ of $M$. We have a Lie group $N\left(\tilde{g}_{0}\right)$ in the similar way to $N\left(g_{0}\right)$. We denote by $\tilde{\mathfrak{T}}$ the sheaf of germs of vector $C^{r+1}$-fields on $\tilde{M}$ and by $\mathfrak{A}\left(\tilde{g}_{0}\right)$ that of infinitesimal automorphisms of $\tilde{g}_{0}$. The Lie algebra of $N\left(\tilde{g}_{0}\right)$ is a subalgebra of $\Gamma(\tilde{\mathfrak{R}}, \tilde{M})$, where $\tilde{\mathfrak{N}}$ is the sheaf of normalizer of $\mathfrak{A}\left(\tilde{g}_{0}\right)$ in $\tilde{\mathfrak{I}}$.

\section{$\S 5$. Deformations of a transitive $G$-structure.}

Definition. Let $g_{t}$ be a 1-parameter family of $G$-structures of class $C^{r}$ parametrized by $t$ of a neighborhood $I$ of 0 in $R$. A family $g_{t}$ is a deformation of $g_{0}$, if there exist an open covering $\left\{U_{i} ; i \in J\right\}$ of $M$ and a family $\left\{f_{i}(x, t) ; i \in J\right\}$ of local continuous transformations of $M \times I$ such that (i) the domain of $f_{i}$ is $U_{i} \times I$, (ii) $f_{i}(x, t)$ for each fixed $t$ is a local $C^{r+1}$-transformation of $M \times t$, (iii) partial derivatives of $f_{i}(x, t)$ of any order $(\leqq r+1)$ with respect to $x$ are continuous on $U_{i} \times I$, (iv) $f_{i}^{*}(x, t)^{-1} g_{0}(f(x, t))=g_{t}(x)$ for $x \in U_{i}$, (v) $f_{i}(0)=$ identity for each $i$ and (vi) $\left\{f_{i}\left(U_{i}, t\right) ; i \in J\right\}$ for each $t$ is an open covering of $M$. Each $G$-structure of a deformation of $g_{0}$ is called to be deformable to $g_{0}$.

Two transitive $\{e\}$-structures are locally equivalent, if they have the same constant structure function (see [6]). When we follow the proof of the above fact, while parametrizing by $t$, we have

Lemma 2. Let $\left\{\theta^{\alpha}(x, t) ; a \in N\right\}$ be a system of independent continuous 1-forms on $R^{N} \times I$ such that each 1 -form is of class $C^{r^{\prime}}$ on $R^{N} \times t$ for each $t$, partial derivatives of $\theta^{\alpha}(x, t)$ of any order $\left(\leqq r^{\prime}\right)$ with respect to $x$ are continuous on $R^{N} \times I,\left\langle\theta^{\alpha}, \frac{\partial}{\partial t}\right\rangle=0$ and $c_{\beta \gamma}^{\alpha}$ are constant, where $d_{x} \theta^{\alpha}=\sum_{\beta, \gamma} c_{\beta \gamma}^{\alpha} \theta^{\beta} \wedge \theta^{r}$ and $d_{x}$ is the exterior differentiation with respect to $x$. Then there exist a neighborhood $U$ of each point of $R^{N}$ and a homeomorphism $\phi(x, t)$ of $U \times I$ into $R^{N} \times I$ such that $\phi(x, t)$ 
for any fixed $t$ is a $C^{r^{\prime}+1}$-diffeomorphism of $U \times t$, partial derivatives of $\phi(x, t)$ of any order $\left(\leqq r^{\prime}+1\right)$ with respect to $x$ are continuous on $R^{N} \times I, \phi^{*}(x, t)^{-1} \theta^{\alpha}(\theta(x, t), 0)=\theta^{\alpha}(x, t)$ and $\phi(x, 0)=i d e n t i t y$.

Since $g_{0}$ is transitive, the $k$-th prolongation of $g_{0}$ is an $\{e\}$-structure with a constant structure function, where $k$ is the order of $G$. Let $\mathscr{D}$ denote the subspace of $\mathcal{G}$ consisting of $G$-structures of which $k$-th prolongations have the same constant functions as that of $g_{0}$.

Proposition 13. A deformation of $g_{0}$ is a continuous mapping $g(t)$ of a neighborhood $I$ of 0 in $R$ into $\mathscr{D}$ with $g(0)=g_{0}$ and conversely.

Proof. From (ii) and (iii), a correspondence $x \rightarrow j_{x}^{r}\left(\bar{f}_{i}(t) g_{0}\right)$ for $x \in U_{i}$ defines a continuous cross-section of $J^{r}(F(M) / G) \times I$ over $U_{i} \times I$, by the application of arguments in Proof of Proposition 7 on $f_{i}(t) U_{i}$, where $\left\{f_{i}, U_{i} ; i \in J\right\}$ defines the deformation $g_{t}$ and

$$
\left(\bar{f}_{i}(t) g_{0}\right)(x)=f_{i}^{*}(x, t)^{-1} g_{0}\left(f_{i}(x, t)\right) .
$$

Since $\bar{f}_{i}(t) g_{0}=g_{t}, j^{r} g_{t}$ is a continuous section of $J^{r}(F(M) / G) \times I$. Therefore $g_{t}$ is a curve in $\mathcal{G}$. Since $g_{t}$ is locally equivalent to $g_{0}$, each $g_{t}$ has the same structure function as $g_{0}$. Therefore $g_{t}$ is a curve in $\mathscr{D}$ through $g_{0}$. Conversely, let $\left\{V_{i} ; i \in J\right\}$ be an open covering of $M$ such that the restriction of the bundle $F(M)$ on each $V_{i}$ are the product $V_{i} \times G L(n)$. Since a curve $g(t)$ in $\mathscr{D}$ through $g_{0}$ is an 1parameter family of $G$-structures continuously dependent to $t$ and $k<r$ by remark of $\S 1$, a set of the portions of the manifolds of $k$-th prolongations of $g(t)$ on $V_{i}$ for all $t \in I$ constructs a domain $V_{i} \times V \times I$ on $R^{N} \times I$, where $N$ is the dimension of the manifold of $k$-th prolongation, and the $\{e\}$-structures of $k$-th prolongations of $g(t)$ construct a system $\left\{\theta^{\alpha}(x, t) ; \alpha \in N\right\}$ of 1 -forms which satisfies the condition of Lemma 2 with $r^{\prime}=r-k$. By Lemma 2, we have a neighborhood $U_{x}\left(\subset V_{i}\right)$ of each point $x$ of $V_{i}$ and a homeomorphism $\phi_{x}^{\prime}\left(x^{\prime}, t\right)$ of $U_{x} \times V \times I$ into $R^{N} \times I$ such that they satisfy the condition of the 
conclusion of Lemma 2. Since local automorphisms of the prolongation of the $G$-structure induce those of the $G$-structure, $\phi_{x}^{\prime}\left(x^{\prime}, t\right)$ induces a diffeomorphism $\phi_{x}\left(x^{\prime \prime}, t\right)$ of $U_{x} \times I$ into $V_{i} \times I$ such that $\phi_{x}\left(x^{\prime \prime}, t\right)$ is a $C^{r+1}$-diffeomorphism of $U_{x} \times t$ into $V_{i} \times t$ for any fixed $t$, partial derivatives of $\phi_{x}\left(x^{\prime \prime}, t\right)$ of any order $(\leqq r+1)$ with respect to $x^{\prime \prime}$ are continuous on $U_{x} \times I$ and $\phi_{x}^{*}\left(x^{\prime \prime}, t\right)^{-1} g_{0}\left(\phi_{x}\left(x^{\prime \prime}, t\right)\right)=g(t)\left(x^{\prime \prime}\right)$. Then for a suitable index $J,\left\{U_{\lambda} ; \lambda \in J\right\}$ and $\left\{\phi_{x_{\lambda}}(x, t) ; \lambda \in J\right\}$ define a deformation $g_{t}$ of $g_{0}$ such that $g(t)=g_{t}$. This fact holds good, even if we use any one of $g(t)$ in place of $g_{0}$. Then we have Propostion, extending the above proof on $I$ successively.

Two deformations $g_{t}^{1}$ and $g_{t}^{2}$ of $g_{0}$ is said to have the same germ of deformation at 0 if there exists a positive number $t_{0}$ such that $g_{t}^{1}=g_{t}^{2}$ on $\left(-t_{0}, t_{0}\right)$. If there is a positive number $t_{0}^{\prime}$ and a continuous family $\left\{f_{t} ; t \in\left(-t_{0}^{\prime}, t_{0}^{\prime}\right)\right\}$ in $\operatorname{Diff}{ }^{(r+1)}(M)$ through $e=f_{0}$ such that $\bar{f}_{t} g_{t}^{1}=g_{t}^{2}$ for any $t \in\left(-t_{0}^{\prime}, t_{0}^{\prime}\right)$, the germ of $g_{t}^{1}$ at 0 is said to be equivalent to that of $g_{t}^{2}$. Thus we have the equivalence class of germs of deformations. Let $\phi(x, t)$ be a local transformation of $M \times I$ such that $\phi(x, 0)$ is identity and $\phi(x, t)$ for any fixed $t$ is a local automorphism of $g_{0}$. Let $\left[A\left(g_{0}\right) \times t\right]$ denote the whole of germs of such $\phi(x, t)$ at every point of $M \times 0$. Then $\left[A\left(g_{0}\right) \times t\right]$ is a sheaf of group on $M$ and we have the 1-chomology set $H^{1}\left(M,\left[A\left(g_{0}\right) \times t\right]\right)$. It is well known that $H^{1}\left(M,\left[A\left(g_{0}\right) \times t\right]\right)$ is one-to-one correspondent to the whole of equivalence classes of germs of deformations of $g_{0}$ (see [3] or [7]).

\section{§6. G-structures having the same infinitesimal automorphisms}

Let $N_{e}\left(\tilde{g}_{0}\right)\left(\operatorname{resp} . A_{e}\left(\tilde{g}_{0}\right)\right)$ be the $e$-component of $N\left(\tilde{g}_{0}\right)\left(\operatorname{resp} . A\left(\tilde{g}_{0}\right)\right)$. On the notation and the argument of $\S 4$, a $G$-structure $\overline{\tilde{f}} \tilde{g}_{0}$ on $\widetilde{M}$ for $\tilde{f} \in N_{e}\left(\tilde{g}_{0}\right)$ has the same infinitesimal automorphisms as $\tilde{g}_{0}$. By Proposition 6, there exists an element a of $N$ such that $\overline{\tilde{f}} \tilde{g}_{0}=\tilde{g}_{0} \cdot a$. If $\overline{\tilde{f}} \tilde{g}_{0}=\tilde{g}_{0} \cdot a=\tilde{g}_{0} \cdot a^{\prime}$, then $a \cdot a^{\prime-1} \in G$. Thus we have a mapping $\sigma: N_{e}$ $\left(\tilde{g_{0}}\right) \rightarrow N / G$ defined by $\sigma(\tilde{f})=q^{\prime}(a)$ where $\overline{\tilde{f}} \tilde{g}_{0}=\tilde{g}_{0} \cdot a$ and $q^{\prime}: N \rightarrow N / G$. 
Proposition 14. The mapping $\sigma$ is an anti-homomorphism and of class $C^{\infty}$ from the Lie group $N_{e}\left(\tilde{g}_{0}\right)$ into $N / G$.

Proof. Since $\left(\tilde{\tilde{f}}_{1} \cdot \tilde{f}_{2}\right) \tilde{g}_{0}=\tilde{\tilde{f}}_{1}\left(\tilde{\tilde{f}}_{2} \tilde{g}_{0}\right)=\left(\tilde{g}_{0} \cdot a_{2}\right) \cdot a_{1}=\tilde{g}_{0} \cdot\left(a_{2} a_{1}\right)$, the mapping $\sigma$ is an anti-homomorphism. Since $N_{e}\left(\tilde{g}_{0}\right)$ is a Lie transformation group of $\tilde{M}$, the correspondence $\tilde{f} \rightarrow \tilde{f}(\tilde{x})$ for a fixed $\tilde{x} \in \tilde{M}$ defines a $C^{\infty}$-mapping $\gamma: N_{e}\left(\tilde{g}_{0}\right) \rightarrow \tilde{M}$. Moreover, $N_{e}\left(\tilde{g}_{0}\right)$ is a Lie transformation group of $F(\tilde{M}) / G$, that is, the correspondence $y \times \tilde{f} \rightarrow \tilde{f}^{*}(y)$ defines a $C^{\infty}$-mapping of $F(\tilde{M}) / G \times N_{e}\left(\tilde{g}_{0}\right)$ into $F(\tilde{M}) / G$, where $\tilde{f}^{*}$ is a transformation of $F(\tilde{M}) / G$ induced by $\tilde{f}$. Since $\tilde{f}(\gamma(\tilde{f}))=\tilde{x}$, the composed mapping

$$
\sigma^{\prime}: \tilde{f} \longrightarrow \gamma(\tilde{f}) \longrightarrow \tilde{g}_{0}(\gamma(\tilde{f})) \longrightarrow \tilde{f}^{*-1}\left(\tilde{g}_{0}(\gamma(\tilde{f}))\right)
$$

is of class $C^{\infty}$ from $N_{e}\left(\tilde{g}_{0}\right)$ into the fibre $F(\tilde{M}) / G \mid \tilde{x}$ of $F(\tilde{M}) / G$ over $\tilde{x}$. By the right translation of $F(\tilde{M}) / G \mid \tilde{x}$ by $N$, we have an imbedding $\nu \tilde{x}$ of $N / G$ into $F(\tilde{M}) / G \mid \tilde{x}$ such that $\nu \tilde{x} q^{\prime}(a)=\tilde{g}_{0}(\tilde{x}) \cdot a$ for $a \in N$. If $\overline{\tilde{f}} \widetilde{g}_{0}=\tilde{g}_{0} \cdot a$. we have

$$
\sigma^{\prime}(\tilde{f})=\tilde{f}^{*-1}\left(\tilde{g}_{0}(\tilde{f}(\tilde{x}))\right)=\tilde{\tilde{f}} \tilde{g}_{0}(\tilde{x})=\tilde{g}_{0}(\tilde{x}) \cdot a=\nu \tilde{x} q^{\prime}(a) .
$$

Then we have a $C^{\infty}$-mapping $\nu \overline{\tilde{x}}^{1} \sigma^{\prime}$ of $N_{e}\left(\tilde{g}_{0}\right)$ into $N / G$, which is $\sigma$.

Proposition 15. For each $\tilde{f} \in N_{e}\left(\tilde{g}_{0}\right)$, the G-structure $\bar{\rho}_{g_{0}} \cdot \sigma(\tilde{f})$ is deformable to $g_{0}$ and has the same infinitesimal automorphisms as $g_{0}$, where $\bar{\rho}_{g_{0}}$ is the $C^{\infty}$-imbedding of $N / G$ into $\mathcal{G}$ in $\S 3$.

Proof. Since $N_{e}\left(\tilde{g}_{0}\right)$ is arcwise connected, we have a curve $\tilde{f}(t)$ of $N_{e}\left(\tilde{g}_{0}\right)$ for $t$ of an interval $I$ such that $\tilde{f}(0)=$ identity and $\tilde{f}\left(t_{0}\right)=\tilde{f}$ for some $t_{0}$. There exists an open neighborhood $\tilde{U}_{\tilde{x}}$ of each $\tilde{x} \in \tilde{M}$ such that the covering mapping $p: \tilde{M} \rightarrow M$ is diffeomorphic on $\tilde{f}(t)$ $\left(\tilde{U}_{\tilde{x}}\right)$ for every $t \in I$. Then the correspondence $(p(y), t) \rightarrow(p(\tilde{f}(t) y), t)$ is a continuous transformation $f_{\tilde{x}}(x, t)$ of an open neighborhood $p\left(\tilde{U}_{\tilde{x}}\right) \times I$ into $M \times I$ such that $f_{\tilde{x}}(x, t)$ for any fixed $t$ is a local $C^{r+1}$. transformation of $M \times t$ and a system $\left\{f_{\tilde{x}}\left(p\left(\tilde{U}_{\tilde{x}}\right) \times I ; \tilde{x} \in \tilde{M}\right\}\right.$ is an 
open covering of $M \times I$. If $f_{\tilde{x}}(p(\tilde{U} \tilde{x}) \times t) \cap f_{\tilde{x}},\left(p\left(\tilde{U}_{\tilde{x}^{\prime}}\right) \times t\right)=V \neq \phi$ a diffeomorphism $f_{\tilde{x}}(x, t)^{-1} \tilde{x}_{\tilde{x}^{\prime}}(x, t)$ is a local automorphism of $g_{0}$ on $\left(f_{\tilde{x}}\right)^{-1} V$, because $\overline{\tilde{f}}(t) \tilde{g}_{0}=\tilde{g}_{0} \cdot a(t)=\bar{p}\left(g_{0} \cdot a(t)\right)$ and then $\left(\bar{f}_{\tilde{x}} g_{0}\right)(V)$ $=\left(\bar{f}_{\tilde{x}^{\prime}} g_{0}\right)(V)=\left(g_{0} a(t)\right)(V)$ where $\sigma(f(t))=q^{\prime}(a(t))$. Since $M$ is compact, there exists a finite index $J$ such that $\left\{p\left(\tilde{U}_{\tilde{x}_{j}}\right) ; j \in J\right\}$ and $\left\{f_{\tilde{x}_{j}} ; j \in J\right\}$ satisfy the conditions of definition of deformations. Since $\bar{f}_{\tilde{x}_{j}} g_{0}=\left(g_{0} \cdot a(t)\right)\left(p\left(\tilde{U}_{\tilde{x}_{j}}\right) \times t\right)=\left(\bar{\rho}_{g_{0}} \sigma(\tilde{f}(t))\right) \mid\left(p\left(\tilde{U}_{\tilde{x}_{j}}\right) \times t\right)$, the family $\bar{\rho}_{g_{0}} \cdot \sigma(\tilde{f}(t))$ is a deformation of $g_{0}$. Since each $G$-structure of $\bar{\rho}_{g_{0}} \cdot \sigma(\tilde{f}(t))$ is associated to $g_{0}$, it has the same infinitesimal automorphisms as $g_{0}$.

By the condition of $g_{0}$ in Introduction, $H^{0}\left(\tilde{M}, \mathfrak{A}\left(\tilde{g}_{0}\right)\right)$ is the Lie algebra of the Lie group $A\left(\tilde{g}_{0}\right)$ of automorphisms of $\tilde{g}_{0}$. Then, if $\tilde{f}_{U}(t)$ is a 1-parameter family of local automorphisms such that $\tilde{f}_{U}(t)(\tilde{x})$ is continuous on $\tilde{U} \times I$ and $\tilde{f}_{\tilde{U}}(0)$ is identity, each of $\tilde{f}_{\tilde{U}}(t)$ can be extended to a unique element of $A_{e}\left(\tilde{g}_{0}\right)$. Let $\tilde{g}$ be a $G$-structure locally equivalent to $\tilde{g}_{0}$. Let $\tilde{\psi}_{\tilde{U}}$ be a local bi- $G$-mapping of $\tilde{g}$ into $\tilde{g}_{0}$ on an open neighborhood $\tilde{U}$, satisfying the condition that there exists an 1-parameter family $\tilde{\psi}_{\tilde{U}}(t)$ of local bi-G-mappings such that $\tilde{\psi}_{\tilde{U}}(t)(\tilde{x})$ is continuous on $\tilde{U} \times I, \tilde{\psi}_{\tilde{U}}(1)=\tilde{\psi}_{\tilde{U}}$ and $\tilde{\psi}_{\tilde{U}}(0)$ is identity. Then the germ of a local bi- $G$-mapping at any $y$ of $\tilde{U}$, satisfying the similar condition to $\psi_{\tilde{U}}$, is the germ of $\tilde{f} \tilde{\psi}_{\tilde{U}}$ at $y$ for some $\tilde{f}$ of $A_{e}\left(\tilde{g}_{0}\right)$. Therefore the portion of the sheaf of germs of local bi- $G$-mapping which satisfies the above condition on $\tilde{U}$, is isomorphic to $\tilde{U} \times A_{e}\left(\tilde{g}_{0}\right)$. Since $\widetilde{M}$ is simly connected, the $\tilde{\psi}_{\tilde{U}}$ can be extended to a global $G$-mapping of $\tilde{g}$ into $\tilde{g}_{0}$.

Proposition 16. If $\tilde{g}_{t}$ is a deformation of $\tilde{g}_{0}$ such that $A\left(\tilde{g}_{t}\right)$ $=A\left(\tilde{g}_{0}\right)$ for each $t$, then $\tilde{g}_{t}$ is trivial.

Proof. There exists a continuous mapping $\tilde{\psi}_{\tilde{U}}(t)$ of $\tilde{U} \times I$ into $\tilde{M}$ for some $\tilde{U}$ such that for each fixed $t, \tilde{\psi}_{\tilde{U}}(t)$ is a local diffeomorphism of $\tilde{U}$ into $\widetilde{M}$ and a bi-G-mapping of $\tilde{g}_{t}$ into $\tilde{g}_{0}$ on $\tilde{U}$. Then $\tilde{\psi}_{\tilde{U}}(t)$ can be extended to a continuous mapping $\tilde{\psi}(t)$ of $\tilde{M} \times I$ in $\widetilde{M}$ such that for each $t, \tilde{\psi}(t)$ is a $G$-mapping of $\tilde{g}_{t}$ into $\tilde{g}_{0}$. Because $A\left(\tilde{g}_{0}\right)=A\left(\tilde{g}_{t}\right)$ and $\tilde{g}_{t}$ satisfies the condition of $\tilde{g}_{0}$, we have a $G$-mapping $\tilde{\psi}^{\prime}(t)$ such that 
$\tilde{\psi}^{\prime}(t) \mid U=\tilde{\psi}_{\tilde{U}}(t)^{-1}$. Since $\tilde{\psi}(t) \cdot \tilde{\psi}^{\prime}(t): \widetilde{M} \rightarrow \widetilde{M}$ is a $G$-mapping and $\tilde{\psi}(t)$ $\tilde{\psi}^{\prime}(t) \mid U=$ identity, $\tilde{\psi}(t) \tilde{\psi^{\prime}}(t)=$ identity, that is, $\tilde{\psi}(t)$ is a diffeomorphism of $\widetilde{M}$ such that $\bar{\psi}(t) \tilde{g}_{0}=\tilde{g}_{t}$. Therefore $\tilde{g}_{t}$ is trivial.

We denote by $\mathcal{S}$ the $g_{0}$-component of the space of $G$-structures which are deformable to $g_{0}$ and have the same infinitesimal automorphisms as $g_{0}$, that is, the $g_{0}$-component of $\mathscr{A} \cap \mathscr{D}$.

Proposition 17. The $C^{\infty}$-mapping $\bar{\rho}_{g_{0}} \sigma(=\mu)$ maps $N_{e}\left(\tilde{g}_{0}\right)$ on $\mathcal{S}$ and the differential $d \mu$ of $\mu$ at e satisfies a formula $\dot{p}(d \mu) \tilde{n}=\mathcal{L}_{\tilde{n}} \tilde{g}_{0}$ for $\tilde{n} \in \Gamma\left(\mathfrak{n}\left(\tilde{g}_{0}\right), \tilde{M}\right)$, where $p$ is the mapping from $\Gamma\left(V_{g_{0}}(F(M) / G)\right)$ onto $\Gamma\left(V_{\tilde{g}_{0}}(F(\tilde{M}) / G)\right)$ induced by $p$ and $\mathfrak{N}\left(\tilde{g}_{0}\right)$ is the sheaf of vector fields of the Lie algebra of $N\left(\tilde{g}_{0}\right)$.

Proof. By Proposition $15, \mu\left(N_{e}\left(\tilde{g}_{0}\right)\right) \subset \mathcal{S}$. For any $g \in \mathcal{S}$, let $g(t)$ be an 1-parameter continuous family in $\mathcal{S}$ for $t \in[0,1]$ such that $g(0)=g_{0}$ and $g(1)=g$. Then the lift $\tilde{g}(t)=\bar{p} g(t)$ of $g(t)$ is a deformation of $\tilde{g}_{0}$ on $\tilde{M}$. By Proposition 16, we have an 1-parameter $\tilde{f}(t)$ of $C^{r+1}$-diffeomorphisms of $\tilde{M}$ such that $\overline{\tilde{f}}(t) \tilde{g}_{0}=\tilde{g}(t)$ and $\tilde{f}(t)(\tilde{x})$ is continuous on $\tilde{M} \times I$. The $G$-structure $\tilde{g}(t)$ for each $t$ has the same infinitesimal automorphisms as $\tilde{g}_{0}$. Since $A(\tilde{g}(t))=A\left(\overline{\tilde{f}}(t) \tilde{g}_{0}\right)=$ $\tilde{f}(t) A\left(\tilde{g}_{0}\right)$, each $\tilde{f}(t)$ transforms $A\left(\tilde{g}_{0}\right)$ onto itself and then $\tilde{f}(t) \in N_{e}\left(\tilde{g}_{0}\right)$. Therefore, $\mu(\tilde{f}(t))=g(t)$ and the image of $\mu$ is $\mathcal{S}$. Moreover, for $\tilde{n} \in \Gamma\left(\mathfrak{R}\left(\tilde{g_{0}}\right), \tilde{M}\right)$ we have

$$
\begin{aligned}
p^{* *} d \mu(\tilde{n})(x) & =\left\{\frac{\mathrm{d}}{\mathrm{d} t} p^{*}\left(g_{0}(x) \cdot a(t)\right)\right\}_{t=0}=\left\{\frac{\mathrm{d}}{\mathrm{d} t}\left(\tilde{g}_{0}(\tilde{x}) \cdot a(t)\right)\right\}_{t=0} \\
& =\left\{\frac{\mathrm{d}}{\mathrm{d} t}\left(\tilde{f}(t) \tilde{g_{0}}\right)(\tilde{x})\right\}_{t=0}=\mathcal{L}_{\tilde{n}} \tilde{g}_{0}(\tilde{x})
\end{aligned}
$$

where $p(\tilde{x})=x, \tilde{f}(t)=\exp t \tilde{n}, \sigma(\tilde{f}(t))=q^{\prime} a(t)$ and $p^{*}\left(\right.$ resp. $\left.p^{* *}\right)$ is the mapping of $F(M) / G$ (resp. $V_{g_{0}}(F(M) / G)$ ) onto $F(\tilde{M}) / G$ (resp. $\left.V_{\tilde{g}_{0}}(F(\tilde{M}) / G)\right)$ induced by $p$. Then $\dot{p}(d \mu(\tilde{n}))=L \tilde{n} \tilde{g} 0$.

Theorem 1. The subspace $\mathcal{S}$ is an immersed submanifold of $\mathcal{G}$. 
Proof. If and only if $\mu\left(\tilde{f}_{i}\right)=\mu\left(\tilde{f}_{2}\right)$ for $\tilde{f}_{1}, \tilde{f}_{2} \in N_{e}\left(\tilde{g}_{0}\right)$, then $\overline{\tilde{f}}_{1} \tilde{g}_{0}=$ $\overline{\tilde{f}}_{2} \tilde{g}_{0}$, that is, $\tilde{f}_{1} \tilde{f}_{2}^{-1} \in A\left(\tilde{g}_{0}\right)$. Now, $N_{e}\left(\tilde{g}_{0}\right) \cap A\left(\tilde{g}_{0}\right)$ is closed in $N_{e}\left(\tilde{g}_{0}\right)$. The differentiable mapping $\mu$ induces a differentiable injection $\bar{\mu}$ from a factor space $N_{e}\left(\tilde{g}_{0}\right) /\left[N_{e}\left(\tilde{g}_{0}\right) \cap A\left(\tilde{g}_{0}\right)\right]$ into the space $\mathcal{G}$. Here, the image of $\bar{\mu}$ is $\mathcal{S}$ and the image of its differential $d \bar{\mu}$ at $e$ is that of $d \mu$, of which the rank is equal to the dimension of $N_{e}\left(\tilde{g}_{0}\right) /\left[N_{e}\left(\tilde{g}_{0}\right) \cap A\left(\tilde{g}_{0}\right)\right]$. Then $d \bar{\mu}$ is injective and split. Therefore $\mathcal{S}$ is an immersed submanifold in $\mathcal{G}$.

Corollary. The tangent space of $\mathcal{S}$ at $g_{0}$ is the vector space $\Gamma\left(\delta_{g_{0}} p^{\prime} \mathfrak{N}\left(\tilde{g}_{0}\right), M\right)$ of all the sections of the subsheaf $\delta_{g_{0}} p^{\prime} \mathfrak{N}\left(\tilde{g}_{0}\right)$ of $V$, where $p^{\prime}$ is the sheaf mapping induced by $p$.

Proof. A diagram of sheaves

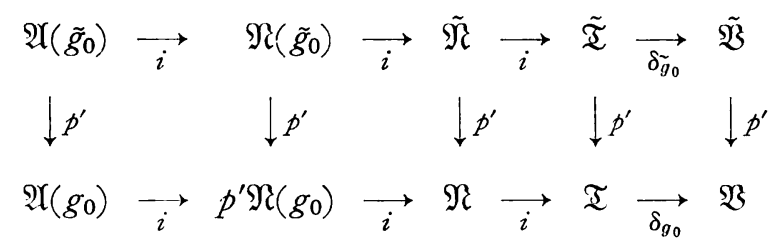

is commutative, where $i$ is the injection. Since $\mathfrak{R}\left(\tilde{g}_{0}\right)$ is a constant sheaf, we have $\Gamma\left(\delta_{\tilde{g}_{0}} \mathfrak{N}\left(\tilde{g}_{0}\right), \tilde{M}\right)=\bar{\delta}_{\tilde{g}_{0}} \Gamma\left(\mathfrak{N}\left(\tilde{g}_{0}\right), \widetilde{M}\right)$. Since $\mathcal{L}_{\tilde{n}} \tilde{g}_{0} \in$ $\bar{\delta}_{\tilde{g}_{0}} \Gamma\left(\mathfrak{N}\left(\tilde{g}_{0}\right), \tilde{M}\right)$ for an $\tilde{n} \in \Gamma\left(\mathfrak{N}\left(\tilde{g}_{0}\right), \tilde{M}\right)$ and $p^{\prime} \delta_{\tilde{g}_{0}} \mathfrak{N}\left(\tilde{g}_{0}\right)=\delta_{g_{0}} p^{\prime} \mathfrak{N}\left(\tilde{g}_{0}\right)$, we have $d \mu(\tilde{n}) \in \Gamma\left(\delta_{g_{0}} p^{\prime} \mathfrak{N}\left(\tilde{g}_{0}\right), M\right)$ by Proposition 17 . Conversely, we have $\dot{p} \dot{g} \in \Gamma\left(\bar{\delta}_{\tilde{g}_{0}}\left(\mathfrak{N}\left(\tilde{g}_{0}\right), \widetilde{M}\right)=\bar{\delta}_{g_{0}} \Gamma\left(\mathfrak{R}\left(\tilde{g}_{0}\right), \widetilde{M}\right)\right.$ for an $\tilde{n} \in \Gamma\left(\mathfrak{N}\left(\tilde{g}_{0}\right), \widetilde{M}\right)$, where $\dot{p} \dot{g}$ is the lift of $\dot{g}$ by $p$. Then $\dot{g}=d \mu(\tilde{n})$.

\section{§7. Equivalence of $G$-structures having the same infinitesimal automorphisms.}

Since $M$ is compact, there is a positive number $\epsilon$ such that the covering mapping $p$ is diffeomorphic on each connected component of $p^{-1}\left(U_{\epsilon}(x)\right)$ for the $\epsilon$-neighborhood $U_{\epsilon}(x)$ of any point $x$ of $M$. For this $\epsilon$, each diffeomorphism $f$ belonging to the $\epsilon$-neighborhood $D_{\epsilon}(e)$ 
of $e$ in $\operatorname{Diff}{ }^{(r+1)}(M)$ induces a diffeomorphism $\tilde{f}$ of $\tilde{M}$ such that $p(\tilde{f}(\tilde{x}))=$ $f(p(\tilde{x}))$ for any $\tilde{x} \in \tilde{M}$ and $\tilde{f}(\tilde{x})$ belongs to the same connected component of $p^{-1}\left(U_{\epsilon}(p(\tilde{x}))\right)$ as $\tilde{x}$. The correspondence $f \rightarrow \tilde{f}$ defines a continuous injection $p$ of $D_{\epsilon}(e)$ into the topological group $\operatorname{Diff}(\tilde{M})$ with the compactopen topology, because the topology of $\operatorname{Diff}^{(r+1)}(M)$ is stronger than the compact-open topology. Since the topology of the Lie group $A_{e}\left(g_{0}\right)$ (resp. $N_{e}\left(g_{0}\right)$ ) is the modified compact-open topology (see [5]), the identity component of $A_{e}\left(g_{0}\right) \cap D_{\epsilon}(e)$ (resp. $N_{e}\left(g_{0}\right) \cap D_{\epsilon}(e)$ ) is an open neighborhood of $e$ in $A_{e}\left(g_{0}\right)$ (resp. $N_{e}\left(g_{0}\right)$ ).

The Lie algebra $\vec{A}(\operatorname{resp} . \bar{N})$ of $A\left(\tilde{g}_{0}\right)\left(\operatorname{resp} . N\left(\tilde{g}_{0}\right)\right)$ is $\Gamma\left(\mathfrak{A}\left(\tilde{g}_{0}\right), \tilde{M}\right)$ (resp. $\left.\Gamma\left(\mathfrak{N}\left(\tilde{g}_{0}\right), \tilde{M}\right)\right)$. Let $\dot{N}$ be the lift ' $p(\Gamma(\mathfrak{N}, M))$ of the Lie algebra $\Gamma(\mathfrak{N}, M)$ of $N\left(\tilde{g}_{0}\right)$. Then $\dot{N}$ and $\bar{A}$ are respectively subalgebra of the Lie algebra $\bar{N}$ of $N\left(\tilde{g}_{0}\right)$. Take a complement $\dot{V}$ of the sum $\dot{N}+\bar{A}$ in $\bar{N}$ and a complement $\dot{N}^{\prime}$ of $\bar{A}$ in $\dot{N}+\bar{A}$. Then, $\bar{N}=\dot{V} \oplus \dot{N}^{\prime} \oplus \bar{A}$. Since $A_{e}\left(\tilde{g}_{0}\right)$ is closed in $N_{e}\left(\tilde{g}_{0}\right)$ and $\tilde{p}\left(A_{e}\left(g_{0}\right) \cap D_{\epsilon}(e)\right)$ is locally closed in $N_{e}\left(\tilde{g}_{0}\right)$, we have

Lemma 3. There exist open neighborhoods $\bar{A}_{0}, N_{0}^{\prime}$ and $V_{0}$ of 0 in $\bar{A}, \dot{N}^{\prime}$ and $\dot{V}$ respectively, such that the mapping

$$
\begin{aligned}
\Phi:(a, b, c) \longrightarrow & (\exp a) \cdot(\exp b) \cdot(\exp c) \\
& \text { for } a \in \vec{A}_{0}, b \in N_{0}^{\prime}, c \in V_{0}
\end{aligned}
$$

is a diffeomorphism of $\bar{A}_{0} \oplus N_{0}^{\prime} \oplus V_{0}$ onto an open neighborhood $\tilde{U}$ of $e$ in $N_{\epsilon}\left(\tilde{g}_{0}\right)$ and $\Phi\left(N_{0}^{\prime}+V_{0}\right) \cap A_{e}\left(\tilde{g}_{0}\right)=e$.

Let $\tilde{V}$ denote the submanifold $\left\{\exp \tilde{v} ; \tilde{v} \in \tilde{V}_{0}\right\}$ of $N_{e}\left(\tilde{g}_{0}\right)$. The restriction of $\mu$ on $\tilde{V}$ is an imbedding and then its image $\mu(\tilde{V})$ is a differentiable submanifold of $\mathcal{S}$ which we denote by $\mathcal{C}$.

Proposition 18. If $\bar{f}(t) g(t)$ is a curve in $C$ for a curve $f(t)$ in $\operatorname{Diff}^{(r+1)}(M)$ through $e=f(0)$ and for a curve $g(t)$ in $C$ through $g_{0}=$ $g(0)$, then there exists $t_{0}>0$ such that $f(t)$ for $t \in\left[-t_{0}, t_{0}\right]$ is in $A_{e}\left(g_{0}\right)$. 
Proof. We have curves $\tilde{n}(t)$ and $\tilde{n}^{\prime}(t)$ in $\tilde{V}$ with $\tilde{n}(0)=\tilde{n}^{\prime}(0)=e$ such that $\mu(\tilde{n}(t))=g(t)$ and $\mu\left(\tilde{n}^{\prime}(t)\right)=\bar{f}(t) g(t)$. Since $f(t) \subset D_{\epsilon}(e)$, where $|t|<t_{0}$ for some $t_{0}>0$, we have $\overline{\bar{n}}^{\prime}(t) g_{0}=(\overline{p \bar{f}}(t)) \overline{\tilde{n}}(t) \tilde{g}_{0}$, that is, $\left(\tilde{n}^{\prime}(t)\right)^{-1}(\tilde{p} f(t)) \tilde{n}(t)=\tilde{b}(t) \subset A_{e}\left(\tilde{g}_{0}\right) . \quad$ Then $\tilde{p} f(t)=\tilde{n}^{\prime}(t) \tilde{b}(t) \tilde{n}(t)^{-1} \subset N_{e}\left(\tilde{g}_{0}\right)$. Taking a smaller $t_{0}$ if necessary, we see that the curves $\tilde{p} f(t), \tilde{b}(t), \tilde{n}^{\prime}(t) \tilde{b}(t)$ and $\tilde{n}^{\prime}(t) \tilde{b}(t) \tilde{n}^{\prime}(t)^{-1}=\tilde{b}^{\prime}(t)$ are in $\tilde{U}$. Since $\tilde{p}(f(t)) \tilde{n}(t)=\tilde{n}^{\prime}(t) \tilde{b}(t)=$ $\tilde{b}^{\prime}(t) \tilde{n}^{\prime}(t), \tilde{p}(f(t)) \subset \tilde{U} \cap P\left(D_{\epsilon}(e)\right), \tilde{n}(t) \subset \tilde{V}, \tilde{n}^{\prime}(t) \subset \tilde{V}$ and $\tilde{b}^{\prime}(t) \subset \tilde{U} \cap A_{e}\left(\tilde{g}_{0}\right)$, we have $\tilde{p}(f(t))=\tilde{b}^{\prime}(t)$ and $\tilde{n}(t)=\tilde{n}^{\prime}(t)$ by Lemma 3 . Therefore, $\tilde{p}(f(t)) \subset \tilde{p}\left(A_{e}\left(g_{0}\right) \cap D_{\epsilon}(e)\right)$, that is, $f(t) \subset A_{e}\left(g_{0}\right)$.

Proposition 19. If we take a suitable connected neighborhood $U_{0}$ of $g_{0}$ on $\mathcal{S}$, then for each $g$ of $U_{0}$ there exist a unique $g^{\prime} \in \mathcal{V}$ and an $f \in N_{e}\left(g_{0}\right) \cap D_{\epsilon}(e)$ such that $g=\bar{f}^{\prime}$, and the correspondence $g \rightarrow g^{\prime}$ is a differentiable mapping of $U_{0}$ onto $Q$.

Proof. If we set $U_{0}=\mu(\tilde{U})$, then we have Proposition from the definition of $\tilde{U}$ and by Lemma 3 .

\section{§. Deformations having the equivalent infinitesimal automorphisms.}

Definition. A deformation $g(t)$ of $g_{0}$ is called to have the equivalent infinitesimal automorphisms, if each $g(t)$ have the infinitesimal automorphisms equivalent to those of $g_{0}$, that is, if there exists a continuous curve $\phi(t)$ in $\operatorname{Diff}(r+1)(M)$ through $e$ such that $\phi(0)=e$ and $\phi(t) A\left(g_{0}\right)=A(g(t))$ for each $t$.

The composed mapping $\tau^{\prime}$ of $\sigma: N_{e}\left(\tilde{g}_{0}\right) \rightarrow N / G$ and $\tau:$ Diff ${ }^{(r+1)}(M)$ $\times N / G \rightarrow \mathscr{G}$ is a $C^{\infty}$-mapping of $\operatorname{Diff}{ }^{(r+1)}(M) \times N_{e}\left(\tilde{g}_{0}\right)$ into $\mathcal{G}$. Moreover, $\tau^{\prime}$ defines a $C^{\infty}$-mapping $\tau^{\prime \prime}$ of $\operatorname{Diff}(r+1)(M) \times \mathcal{S}$ into $\mathscr{G}$ by formula $\tau^{\prime \prime}(i d . \times \mu)=\tau^{\prime}$, such that $\tau^{\prime \prime}(f, g)=\bar{f} g$ for $f \in \operatorname{Diff}(r+1)(M)$ and $g \in \mathcal{S}$.

Proposition 20. There exists an open neighborhood $U_{e}$ of $e$ in $\operatorname{Diff}(r+1)(M)$ such that, if and only if a deformation $g(t)$ of $g_{0}$ have 
the equivalent infinitesimal automorphisms, $g^{(}(t)$ is a curve through $g_{0}$ in the image of $U_{e} \times \checkmark$ by the $C^{\infty}$-mapping $\tau^{\prime \prime}$ for $t$ of some neighborhood of 0 in $R$.

Proof. The differential of $\tau^{\prime \prime}$ at $\left(e, g_{0}\right)$ is a continuous linear mapping $\theta+\dot{g} \rightarrow \bar{\delta}_{g_{0}} \theta+\dot{g}$ for $\theta \in T_{e}\left(\operatorname{Diff}{ }^{(r+1)}(M)\right)$ and $\dot{g} \in T_{g_{0}}(\mathcal{S})$. If $\dot{g}$ is tangent to $\mathcal{Q}$, we have $\tilde{v} \in \dot{V}_{0}$ such that $d \mu(\tilde{v})=\dot{g}$ and $\dot{p} \dot{g}=\mathcal{L}_{\tilde{v}} \tilde{g}_{0}$. If $\bar{\delta}_{g_{0}} \theta+\dot{g}=0$, we have

$$
\dot{p} \bar{\delta}_{g_{0}} \theta+\dot{p} \dot{g}=\mathcal{L}^{\prime} p \theta \tilde{g}_{0}+\mathcal{L}_{\tilde{v}} \tilde{g}_{0}=0
$$

and then ' $p \theta \in \bar{A}+\dot{V}$, where ' $p$ denote the lift of vector fields on $M$. Since ' $p \theta \in \dot{N}$, we have ' $p \theta \in \bar{A} \cap N$ and then $\theta \in \Gamma\left(\mathfrak{U}\left(g_{0}\right), M\right)$. Since there exists a closed complement $D$ of $\Gamma\left(\mathfrak{A}\left(g_{0}\right), M\right)$ in $T_{e}\left(\operatorname{Diff}{ }^{(r+1)}(M)\right)$, we have an open neighborhood $U_{e}$ of $e$ on $\operatorname{Diff}{ }^{(r+1)}(M)$ and a submanifold $C$ tangent to $D$ at $e$ in $U_{e}$ such that $\tau^{\prime \prime}\left(U_{e} \times C\right)=\tau^{\prime \prime}(C \times C)$ and $\tau^{\prime \prime}$ is diffeomorphic on $C \times C$. If $g(t)$ is a curve in $\tau^{\prime \prime}(C \times C)$ through $g_{0}$, then we have a curve $f(t)$ in $C$ and a curve $v(t)$ in $C$ such that $g(t)=$ $\bar{f}(t) v(t)$. Therefore,

$$
A(g(t))=A(\bar{f}(t) v(t))=f(t) A(v(t))=f(t) A\left(g_{0}\right),
$$

that is, $g(t)$ is a deformation having the equivalent infinitesimal automorphisms. Conversely, if for a deformation $g(t)$ of $g_{0}$ there exists $f(t)$ such that $A(g(t))=f(t) A\left(g_{0}\right)$ and $f(0)=$ identity, then $A\left(g_{0}\right)=$ $A\left(\bar{f}(t)^{-1} g(t)\right)$. By Theorem 1 and Proposition 19, $\bar{f}(t)^{-1} g(t)$ is a curve $v(t)$ in $Q$ for a sufficiently small $|t|$. Then $f(t)$ is in $U_{e}$ for $t$ of some neighborhood of 0 in $R$ and $g(t)=\bar{f}(t) v(t)$ is in $\tau^{\prime \prime}\left(U_{e} \times C\right)$ ).

Taking germs at $t=0$, the above facts are represented in the cohomology with coefficient sheaf as follows. Let $\left\{f_{i}(t), U_{i} ; i \in J\right\}$ be a system of an open covering $\left\{U_{i}\right\}$ of $M$ and local diffeomorphisms $f_{i}$ defining a deformation $g(t)$ of $g_{0}$. For $i, j \in J$ such that $U_{i} \cap U_{j}$ $\neq \phi$, a local transformation $f_{i}(t)^{-1} f_{j}(t)$ is considered as a 1-parameter family of local automorphisms of $g_{0}$ continuously dependent to $t$ and its germ at $t=0$ is a section of the sheaf $\left[A\left(g_{0}\right) \times t\right]$ over $U_{i} \cap U_{j}$. 
Let $\psi(x, t)$ be a local transformation of $M \times I$ such that $\psi(x, 0)$ is identity and $\psi(x, t)$ for any fixed $t$ is a local $C^{r+1}$-transformation of $M \times t$ which transforms $\mathfrak{A}\left(g_{0}\right)$ onto itself and such that partial derivatives of $\psi(x, t)$ of any order $(\leqq r+1)$ with respect to $x$ are continuous on $M \times I$. Let $\left[N\left(g_{0}\right) \times t\right]$ denote the whole of germs of such local transformations at every point of $M \times 0$. Then $\left[N\left(g_{0}\right) \times t\right]$ is a sheaf of group and $\left[N\left(g_{0}\right) \times t\right] \supset\left[A\left(g_{0}\right) \times t\right]$. Therefore, a system

\{germs of $f_{i}(t)^{-1} f_{j}(t)$ at $t=0 ; i, j \in J$ such that $\left.U_{i} \cap U_{j} \neq \phi\right\}$

is a $\left[N\left(g_{0}\right) \times t\right]$-valued 1-cocycle of the nerve of $\left\{U_{i}\right\}$. This cocycle is coboudary, if and only if the germ of $g(t)$ is equivalent to a deformation having the equivalent infinitesimal automorphisms. Let $\Omega$ denote the correspondence

$$
H^{1}\left(M,\left[A\left(g_{0}\right) \times t\right]\right) \longrightarrow H^{1}\left(M,\left[N\left(g_{0}\right) \times t\right]\right)
$$

induced by the injection $A\left(g_{0}\right) \rightarrow N\left(g_{0}\right)$. Then we have

Theorem 2. A cohomology class $g$ of $H^{1}\left(M,\left[A\left(g_{0}\right) \times t\right]\right)$ corresponds to a class of germ of a deformation having the equivalent infinitesimal automorphisms, if and only if $\Omega \cdot g$ is coboundary in $H^{1}(M$, $\left.\left[N\left(g_{0}\right) \times t\right]\right)$. Any such class is represented by a unique germ of a curve in $Q$.

Since $\left.d \tau^{\prime \prime}\left(T_{e}(C)+T_{g_{0}}(C)\right)=\left\{\bar{\delta}_{g_{0}} \theta+\dot{v} ; \theta \in T_{e}(C), \dot{v} \in T_{g_{0}}(C)\right)\right\}$, the tangent vector of a differentiable curve in $\tau^{\prime \prime}\left(U_{e} \times C\right)$ at $t=0$ is $\bar{\delta}_{g_{0}} \theta+\dot{v}$. Conversely, for any $\theta \in T_{e}(C)$ and any $\dot{v} \in T_{g_{0}}(C)$, a vector $\bar{\delta}_{g_{0}} \theta+\dot{v}$ is tangent to a differentiable curve in $\tau^{\prime \prime}(U \times C)$ at $t=0$. Here, from the definition of $C$ and $C$,

$$
\left\{\bar{\delta}_{g_{0}} \theta+\dot{v} ; \theta \in T_{e}(C), \dot{v} \in T_{g_{0}}(C)\right\}=\left\{\bar{\delta}_{g_{0}} \Gamma(\mathfrak{I})+\Gamma\left(\delta_{g_{0}} p^{\prime} \mathfrak{R}\left(\tilde{g}_{0}\right)\right)\right\}
$$

Each element of $\bar{\delta}_{g_{0}} \Gamma(\mathfrak{T})+\Gamma\left(\delta_{g_{0}} p^{\prime} \mathfrak{\Re}\left(\tilde{g}_{0}\right)\right)$ is called an infinitesimal deformation of $g_{0}$ having the equivalent automorphisms. Thus we have 
Theorem 3. Every infinitesimal deformation having the equivalent infinitesimal automorphisms can be extended to a deformation having the equivalent infinitesimal automorphisms.

The whole of equivalent classes of infinitesimal deformations of $g_{0}$ is a linear space $\Gamma\left(\delta_{g_{0}} \mathfrak{I}\right) / \bar{\delta}_{g_{0}} \Gamma(\mathfrak{I})$ which is isomorphic to $H^{1}\left(M, \mathfrak{A}\left(g_{0}\right)\right)$. Since $\mathfrak{A}\left(g_{0}\right) \subset p^{\prime} \mathfrak{N}\left(\widetilde{g}_{0}\right)$, we have a homomorphism $\omega^{\prime}: H^{1}\left(M, \mathfrak{A}\left(g_{0}\right)\right) \rightarrow$ $\left.H^{1}\left(M, p^{\prime}\right) \mathfrak{l}\left(\tilde{g}_{0}\right)\right)$. Ker $\omega^{\prime}(=\mathcal{K}$ in Introduction $)$ is the whole of equivalent classes of infinitesimal deformations having the equivalent infinitesimal automorphisms and this is a linear space with the dimension of the manifold $\mathcal{C}$, which is equal to $\left[\operatorname{dim} . N\left(\tilde{g}_{0}\right)-\operatorname{dim} . A\left(\tilde{g}_{0}\right)-\right.$ $\left.\operatorname{dim} . N\left(g_{0}\right)+\operatorname{dim} . A\left(g_{0}\right)\right]$. Then we have

Theorem 4. If $\omega^{\prime}: H^{1}\left(M, \mathfrak{A}\left(g_{0}\right)\right) \rightarrow H^{1}\left(M, p^{\prime} \mathfrak{N}\left(\widetilde{g}_{0}\right)\right)$ is injective, that is, if $\left[\operatorname{dim} . N\left(\tilde{g}_{0}\right)-\operatorname{dim} . A\left(\tilde{g}_{0}\right)-\operatorname{dim} . N\left(g_{0}\right)+\operatorname{dim} . A\left(g_{0}\right)\right]=0$, then every deformations of $g_{0}$ having the equivalent infinitesimal automorphisms are trivial.

\section{Kyoto University}

\section{References}

[1] D. Bernard, Sur la géométrie différentielle des G-structures, Ann. Inst. Fourier, 10 (1960), 151-270.

[2] J. Eells Jt., On the geometry of function space, Symposium de Topologia Algebrica, Mexico, (1958), 303-307.

[3] P. A. Griffiths, Deformations of G-structures, Math. Ann., 155 (1964), 292-315, 158 (1965), 326-351.

[4] P. A. Griffiths, On the existence of a locally complete germ of deformation of certain G-structures, Math. Ann., 159 (1965), 151-171.

[5] R. S. Palais, A global formulation of the Lie theory of transformation groups, Memoirs of Amer. Math. Soc., No. 22 (1957).

[6] S. Sternberg, Lectures on a differential geometry, Prentice-hall, (1964).

[7] T. Yagyu, On deformations of cross-sections of a differentiable fibre bundle, J. of Math. of Kyoto Univ., 2 (1963), 209-226. 